\title{
Dynamic Structure Models for Scalar Flux and Dissipation in Large Eddy Simulation
}

\author{
Sergei Chumakov* and Christopher J. Rutland ${ }^{\dagger}$ \\ University of Wisconsin-Madison, Madison, Wisconsin 53706
}

\begin{abstract}
A new class of subgrid scale models (dynamic structure models) for large eddy simulation is proposed for subgrid scalar flux and dissipation terms. The structure of the modeled terms is taken from the corresponding Leonard terms by the use of the test filter size equal to the base filter size, and a particular form of the scaling factor is proposed. The models are evaluated using available direct numerical simulation data. The evaluation results compare well with viscosity and similarity models. The dynamic structure models have been found to be robust and to work well under various conditions, including various test-to-base filter size ratios and filter skewnesses. Models for both terms employ the subgrid scalar variance as a part of the scaling factor. It is possible not to model the subgrid variance, but to find it via the evolution equation. A new form of the transport equation for subgrid scalar variance that contains only one unclosed term is presented.
\end{abstract}

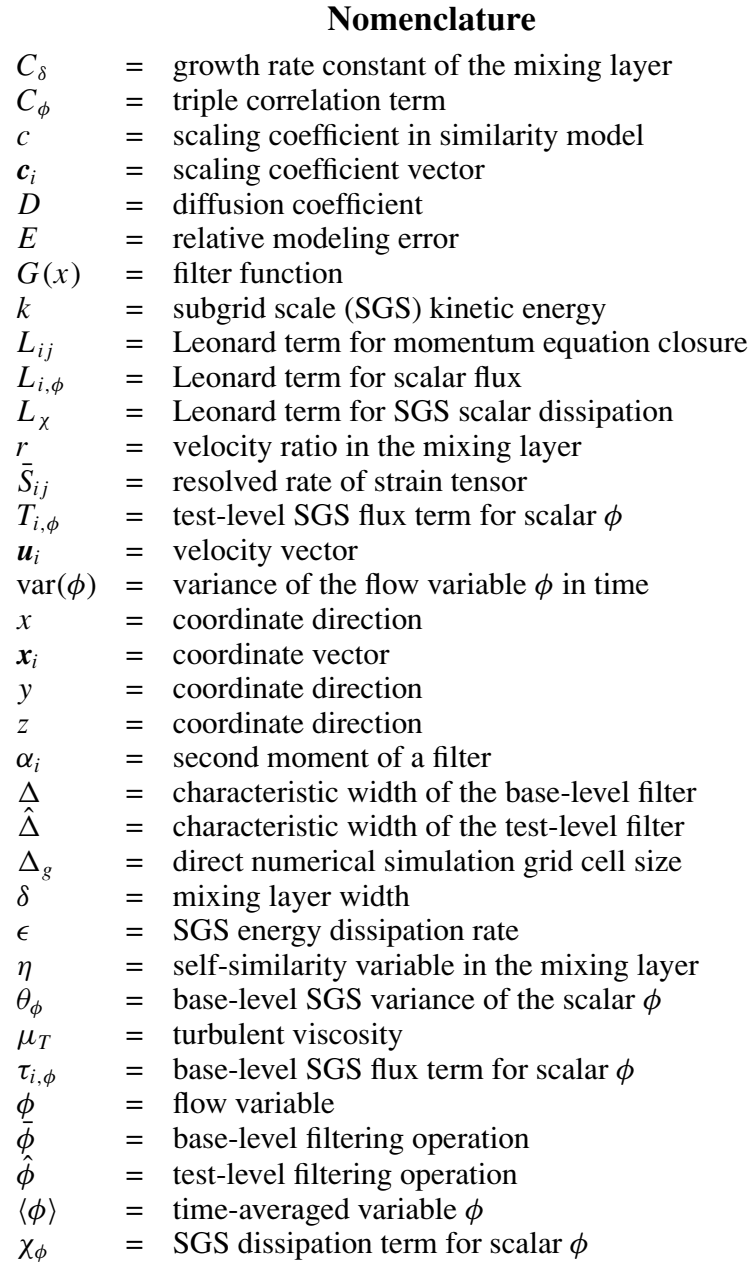

Received 23 December 2002; revision received 23 October 2003; accepted for publication 23 October 2003. Copyright (C) 2004 by Sergei Chumakov and Christopher J. Rutland. Published by the American Institute of Aeronautics and Astronautics, Inc., with permission. Copies of this paper may be made for personal or internal use, on condition that the copier pay the $\$ 10.00$ per-copy fee to the Copyright Clearance Center, Inc., 222 Rosewood Drive, Danvers, MA 01923; include the code 0001-1452/04 \$10.00 in correspondence with the CCC.

${ }^{*}$ Graduate Student, Engine Research Center, Department of Mechanical Engineering; sergei@erc.wisc.edu.

Professor, Engine Research Center, Department of Mechanical Engineering. Senior Member AIAA.
$\begin{array}{ll}\Theta_{\bar{\phi}} & =\text { test-level SGS variance of the scalar } \bar{\phi} \\ \omega & =\text { source term due to chemistry }\end{array}$

\section{Introduction}

$\mathbf{L}$ ARGE eddy simulation (LES) is based on the decomposition of flow variables into resolved (filtered, large-scale) and unresolved [subgrid-scale (SGS)] components. The resolved part is obtained by directly solving the filtered conservation equations, whereas the effect of the unresolved part is modeled. The resolved and unresolved scales are separated with a spatial filtering procedure. For any flow variable $\phi$, it is postulated that $\phi=\bar{\phi}+\phi^{\prime}$, where $\bar{\phi}=\phi * G$ is the resolved part, $\phi^{\prime}=\phi-\bar{\phi}$ is the unresolved part, $*$ denotes convolution operation, and $G$ is called a filter function. The shape and size of $G$ gives rise to the filter size $\Delta$. The most common type of filter function is a box, or top-hat function: $G(x)=\Delta^{-3}$ for $|x|<\Delta / 2$, and $G(x)=0$ otherwise.

Application of the filtering operation to any transport equation gives rise to unclosed terms. Over the course of more than 30 years, a multitude of models has been proposed and evaluated to close the momentum transport equations. ${ }^{1}$ However, only a few models deal with the terms that appear in scalar transport equations.

Consider the following transport equation for a scalar $\phi$ :

$$
\frac{\partial \phi}{\partial t}+\frac{\partial u_{i} \phi}{\partial x_{i}}=\frac{\partial}{\partial x_{i}}\left[D \frac{\partial \phi}{\partial x_{i}}\right]+\omega
$$

where $D$ is the diffusion coefficient and $\omega$ is the source term that may arise, for example, from chemistry. Application of the LES filtering operation yields the LES transport equation

$$
\frac{\partial \bar{\phi}}{\partial t}+\frac{\partial \bar{u}_{i} \bar{\phi}}{\partial x_{i}}=\frac{\partial}{\partial x_{i}}\left[D \frac{\partial \bar{\phi}}{\partial x_{i}}\right]+\bar{\omega}-\frac{\partial \tau_{i, \phi}}{\partial x_{i}}
$$

where $\tau_{i, \phi}=\overline{u_{i} \phi}-\bar{u}_{i} \bar{\phi}$ is the subgrid flux term. The hazards of modeling the source term $\bar{\omega}$ are well known. ${ }^{2,3}$ Fewer models have been developed for $\tau_{i, \phi}$.

The oldest and most popular type of models is the viscosity, or Smagorinsky-type models, named after the pioneering work of Smagorinsky. ${ }^{4}$ The general form of a viscosity model is $\tau_{i, \phi} \approx-\mu_{T}\left(\partial \bar{\phi} / \partial x_{i}\right)$. Various algorithms for calculation of the turbulent viscosity $\mu_{T}$ have been proposed. ${ }^{5,6}$ The recent ones include the dynamic determination of $\mu_{T}$ utilizing the Germano identity ${ }^{7}$ for scalar terms:

$$
L_{i, \phi}=T_{i, \phi}-\widehat{\tau_{i, \phi}}
$$


where $L_{i, \phi}=\widehat{\bar{u}_{i} \bar{\phi}}-\widehat{\hat{u}_{i}} \hat{\bar{\phi}}$ is the Leonard term for scalar flux, $T_{i, \phi}=$ $\widehat{\overline{u_{i} \phi}}-\widehat{\bar{u}_{i}} \phi$ is the test-level subgrid flux term, and superscript denotes the test filtering operation.

A more successful approach is used in similarity models that use the Leonard term itself as a model for certain ratio of characteristic lengths $\Delta$ and $\hat{\Delta}$. This class of models has been shown to perform significantly better than viscosity-type models for the momentum equation closure. ${ }^{8}$

A combined approach also has been investigated. ${ }^{9}$ The term $\tau_{i, \phi}$ was modeled as a linear combination of the Leonard term and Smagorinsky model, which may be viewed as an analog of the linear combination model for momentum equation. ${ }^{10}$ Tests show that this class of models perform better than the similarity model alone. However, a priori knowledge of the flow is required to determine the values of coefficients in the linear combination.

In the field of combustion modeling, considerable effort has been spent in modeling two other scalar-related quantities:

$$
\begin{aligned}
\theta_{\phi} & =\frac{1}{2}(\overline{\phi \phi}-\bar{\phi} \bar{\phi}) \\
\bar{\chi} & =D \overline{\frac{\partial \phi}{\partial x_{i}} \frac{\partial \phi}{\partial x_{i}}}
\end{aligned}
$$

SGS scalar variance and SGS scalar dissipation rate. Although several models can be found in the literature for the SGS scalar variance $\theta_{\phi}$, we consider adding a separate transport equation for $\theta_{\phi}$ to our set of LES equations. The SGS scalar dissipation rate has been modeled in several ways. The most common model is given by application of traditional momentum methods ${ }^{11,12}: \bar{\chi}=C \theta_{\phi} / \tau$, where $\tau$ is the turbulent timescale obtained from momentum equation closure, and $C$ is an a priori constant. This model has been criticized and an alternative, more complex model with three terms has been proposed by Girimaji and Zhou. ${ }^{13}$ The model contained one a priori scaling constant. Subsequently, an effort was made to incorporate a dynamic procedure to determine the constant. ${ }^{14}$ However, in a priori tests with incompressible isotropic turbulence, it was found that the dynamic coefficient led to decreased accuracy relative to the constant coefficient. Determination by the use of an assumed scalar spectrum was attempted later with moderate improvement. ${ }^{15}$

We argue that it seems more appropriate to model the term $\chi_{\phi}$, defined in the following way:

$$
\chi_{\phi}=D\left[\overline{\frac{\partial \phi}{\partial x_{i}} \frac{\partial \phi}{\partial x_{i}}}-\overline{\frac{\partial \phi}{\partial x_{i}}} \overline{\frac{\partial \phi}{\partial x_{i}}}\right]
$$

rather than model $\bar{\chi}$ as it has been in the literature. The form of the suggested model for $\chi_{\phi}$ is motivated by the scale-similarity ideas, and the particular form of the adaptive scaling factor is motivated by the dynamic structure model for the SGS scalar flux term presented later in the paper.

In this work, new models are presented for the SGS scalar flux term $\tau_{i, \phi}$ and SGS scalar dissipation term $\chi_{\phi}$. The models are evaluated a priori with available direct numerical simulation (DNS) data for nonreacting channel flow, Couette flow, and mixing layer. Then, a posteriori evaluation is performed by implementation of the models in an LES code. An LES of a nonreacting incompressible mixing layer was performed, and results were compared to those of DNS.

\section{SGS Scalar Flux}

The main idea of the dynamic approach consists of treating the turbulent eddies as fractal-like structures. Thus, an extrapolation of small-scale statistics is possible, given the knowledge of large-scale fields. This procedure requires another filtering operation, known as test filtering, usually denoted by superscript - .

By analogy with the similarity models for the momentum equation, ${ }^{8}$ we use the assumption of scale invariance in a strong, almost literal sense. The full structure of the scalar field at scales below $\Delta$ is postulated to be similar to the scales above $\Delta$. This, through the Leonard-like terms, provides us with the essential structure of model terms. Thus, the following form of the model is proposed:

$$
\tau_{i, \phi} \approx c L_{i, \phi}
$$

where $L_{i, \phi}$ is the Leonard term for scalar flux described earlier and $c$ is the scaling factor to be determined.

For the momentum equation, the observation that there exists a high degree of correlation between the Leonard term and the corresponding subgrid term was observed for the case of a turbulent jet. ${ }^{16}$ A good correlation was observed between the SGS stress tensor $\tau_{i j}=\overline{u_{i} u_{j}}-\bar{u}_{i} \bar{u}_{j}$ and the Leonard term $L_{i j}=\overline{\bar{u}}_{i} \bar{u}_{j}-\widehat{\bar{u}_{i}} \widehat{\bar{u}_{j}}$, and the model similar to approximation (7) was proposed. It was demonstrated that this model captures backscatter in a reasonable manner and that it scales correctly near solid boundaries. By employment of a test filter size of $\hat{\Delta}=2 \Delta, L_{i j}$ was found to be of the same order of magnitude as $\tau_{i j}$; therefore, $c$ was assumed equal to unity.

For the proposed model form (7), the scaling factor $c$ cannot be readily determined dynamically, that is, with the Germano identity (3), because the procedure becomes recursive. Therefore, an alternative approach must be found.

We propose the use the ratio of the subgrid variances on the base and test level as a scaling factor $c$ in form (7). The motivation for this particular form is that the subgrid variance represents the variance of the filtered probability density function (PDF). ${ }^{17}$ Thus, the subgrid variance of a scalar can be regarded as a measure of degree of resolution of the scalar field. This assumption, together with the assumed scale invariance, yields the new class of LES models. The structure of the unknown subgrid terms is provided by corresponding Leonard terms, and the scaling factor should be determined dynamically instead of being an a priori constant. In this paper, we propose two models of this class, for the scalar subgrid flux and dissipation terms.

The final form of the dynamic structure model for $\tau_{i, \phi}$ can be formulated as follows:

$$
\tau_{i, \phi} \approx\left(\theta_{\phi} / \Theta_{\bar{\phi}}\right) L_{i, \phi}
$$

where $\theta_{\phi}=\frac{1}{2}(\overline{\phi \phi}-\bar{\phi} \bar{\phi})$ is the subgrid variance of $\phi$ on the base level, and $\Theta_{\bar{\phi}}=\frac{1}{2}(\overline{\bar{\phi}} \bar{\phi}-\hat{\bar{\phi}} \hat{\bar{\phi}})$ is the subgrid variance of $\bar{\phi}$ on the test level.

Note that this model can be derived formally via the Germano identity if, instead of the general form (7), we postulate

$$
\tau_{i, \phi} \approx c_{i} \theta_{\phi}, \quad T_{1, \phi} \approx c_{i}(\widehat{\overline{\phi \phi}}-\hat{\bar{\phi}} \hat{\bar{\phi}})
$$

The assumption that $c_{i}$ varies slowly with space allows us to remove $\boldsymbol{c}_{i}$ from under the convolution integrals. Then, we can use Germano identity (3) to determine that $\boldsymbol{c}_{i}=L_{i, \phi} / \Theta_{\bar{\phi}}$, similar to dynamic structure models for the momentum equations. ${ }^{18}$

\section{SGS Scalar Variance and Dissipation}

The subgrid variance $\theta_{\phi}$ cannot be determined from the resolved field and, thus, has to be modeled. An extensive amount of work has been conducted in the area of modeling $\theta_{\phi}$ directly due to its importance in the LES of the reacting flows., ${ }^{2,3,19,20}$ As an alternative, it is possible to derive a transport equation for $\theta_{\phi}$ (Ref. 12):

$$
\begin{gathered}
\frac{\partial \theta_{\phi}}{\partial t}+\frac{\partial \bar{u}_{i} \theta_{\phi}}{\partial x_{i}}=\frac{\partial}{\partial x_{i}}\left[D \frac{\partial \theta_{\phi}}{\partial x_{i}}\right]-\chi_{\phi}-C_{\phi}+\bar{\phi} \frac{\partial \tau_{i, \phi}}{\partial x_{i}} \\
C_{\phi}=\frac{1}{2} \frac{\partial}{\partial x_{i}}\left[\overline{\phi^{2} u_{i}}-\overline{\phi^{2}} \bar{u}_{i}\right], \quad \chi_{\phi}=D\left[\overline{\frac{\partial \phi}{\partial x_{i}} \frac{\partial \phi}{\partial x_{i}}}-\overline{\frac{\partial \phi}{\partial x_{i}}} \frac{\overline{\partial \phi}}{\partial x_{i}}\right]
\end{gathered}
$$

where the triple correlation term $C_{\phi}$ and the subgrid dissipation term $\chi_{\phi}$ have to be modeled again. This is probably the reason why the subgrid variance evolution equation is rarely used in the literature, whereas the preference is given to separate modeling of subgrid variance and scalar dissipation. ${ }^{20}$ 
Note that our definition of subgrid variance and dissipation differ from those found in the literature, where they are defined as $\overline{\phi^{2}}-\bar{\phi}^{2}$ and $\overline{\left(\partial \phi / \partial x_{i}\right)\left(\partial \phi / \partial x_{i}\right)}$, respectively. The form of $\theta_{\phi}$ is chosen to correspond to the form of SGS kinetic energy $k=\frac{1}{2}\left(\overline{u_{i} u_{i}}-\bar{u}_{i} \bar{u}_{i}\right)$, and the definition (11) is argued to reflect the effect of scalar dissipation on scales less than $\Delta$ appropriately.

We can deal with the triple correlation term in Eq. (10) by using the following series expansion ${ }^{21}$ :

$$
\overline{f g}-\bar{f} \bar{g}=2 \alpha_{[k]} \frac{\partial \bar{f}}{\partial x_{k}} \frac{\partial \bar{g}}{\partial x_{k}}+\mathcal{O}\left(\alpha_{k}^{2}\right)
$$

Square brackets indicate that the indices do not participate in summation. Quantities $\alpha_{k}$ are defined as

$$
\alpha_{k}=\frac{1}{2} \int_{-\infty}^{\infty} x_{k}^{2} G(x) \mathrm{d} x_{k}
$$

If we apply the expansion (12) to the triple correlation term $C_{\phi}$, we obtain the following:

$$
\begin{aligned}
C_{\phi} \equiv & \frac{1}{2} \frac{\partial}{\partial x_{i}}\left[2 \alpha_{[k]} \frac{\partial \bar{u}_{i}}{\partial x_{k}} \frac{\partial \bar{\phi}}{\partial x_{k}}+\mathcal{O}\left(\alpha_{k}^{2}\right)\right] \\
& =\frac{1}{2} \frac{\partial}{\partial x_{i}}\left[2 \alpha _ { [ k ] } \frac { \partial \overline { u } _ { i } } { \partial x _ { k } } \frac { \partial } { \partial x _ { k } } \left(\bar{\phi} \bar{\phi}+2 \alpha_{[m]} \frac{\partial \bar{\phi}}{\partial x_{m}} \frac{\partial \bar{\phi}}{\partial x_{m}}\right.\right. \\
& \left.\left.+\mathcal{O}\left(\alpha_{m}^{2}\right)\right)+\mathcal{O}\left(\alpha_{k}^{2}\right)\right] \\
& =\frac{1}{2} \frac{\partial}{\partial x_{i}}\left[2 \alpha_{[k]} \frac{\partial \bar{u}_{i}}{\partial x_{k}} \frac{\partial \bar{\phi} \bar{\phi}}{\partial x_{k}}+\mathcal{O}\left(\alpha_{k}^{2}\right)\right] \\
& =\frac{\partial}{\partial x_{i}}\left[\bar{\phi} \cdot 2 \alpha_{[k]} \frac{\partial \bar{u}_{i}}{\partial x_{k}} \frac{\partial \bar{\phi}}{\partial x_{k}}+\mathcal{O}\left(\alpha_{k}^{2}\right)\right] \\
& =\bar{\phi} \cdot \frac{\partial}{\partial x_{i}}\left[2 \alpha_{[k]} \frac{\partial \bar{u}_{i}}{\partial x_{k}} \frac{\partial \bar{\phi}}{\partial x_{k}}\right]+\frac{\partial \bar{\phi}}{\partial x_{i}} \cdot 2 \alpha_{[k]} \frac{\partial \bar{u}_{i}}{\partial x_{k}} \frac{\partial \bar{\phi}}{\partial x_{k}}+\mathcal{O}\left(\alpha_{k}^{2}\right)
\end{aligned}
$$

Now we combine the obtained expansion with one for the last term on the right-hand side of Eq. (10) to obtain the following:

$$
\begin{gathered}
\bar{\phi} \cdot \frac{\partial \tau_{i, \phi}}{\partial x_{i}}-C_{\phi}=\bar{\phi} \frac{\partial}{\partial x_{i}}\left[2 \alpha_{[k]} \frac{\partial \bar{\phi}}{\partial x_{k}} \frac{\partial \bar{u}_{i}}{\partial x_{k}}+O\left(\alpha_{k}^{2}\right)\right]-C_{\phi} \\
=-\frac{\partial \bar{\phi}}{\partial x_{i}} \cdot 2 \alpha_{[k]} \frac{\partial \bar{u}_{i}}{\partial x_{k}} \frac{\partial \bar{\phi}}{\partial x_{k}}+\mathcal{O}\left(\alpha_{k}^{2}\right)=-\frac{\partial \bar{\phi}}{\partial x_{i}} \cdot \tau_{i, \phi}+\mathcal{O}\left(\alpha_{k}^{2}\right)
\end{gathered}
$$

Thus, Eq. (10) becomes

$$
\frac{\partial \theta_{\phi}}{\partial t}+\frac{\partial \bar{u}_{i} \theta_{\phi}}{\partial x_{i}}=\frac{\partial}{\partial x_{i}}\left[D \frac{\partial \theta_{\phi}}{\partial x_{i}}\right]-\chi_{\phi}-\tau_{i, \phi} \frac{\partial \bar{\phi}}{\partial x_{i}}+\mathcal{O}\left(\Delta^{4}\right)
$$

Note that Eq. (10) is exact, whereas Eq. (13) contains modeling assumptions. Moreover, the subgrid scalar dissipation $\chi_{\phi}$ still has to be modeled.

By analogy with approximation (8), we propose the use of the following model for dissipation:

$$
\chi_{\phi} \approx 2\left(\theta_{\phi} / \Theta_{\phi}\right) L_{\chi}
$$

where

$$
L_{\chi}=D\left[\frac{\partial \widehat{\bar{\phi}}}{\partial x_{i}} \frac{\partial \bar{\phi}}{\partial x_{i}}-\widehat{\frac{\partial \bar{\phi}}{\partial x_{i}}} \frac{\partial \bar{\phi}}{\partial x_{i}}\right]
$$

is the Leonard term for subgrid dissipation.

In the remainder of the paper, models (8) and (14) shall be referred to as dynamic structure (DS) models, following the original work ${ }^{18}$ for subgrid momentum stresses.

\section{A Priori Evaluation}

To evaluate the proposed model a priori, data from two types of DNS were used.

First, data from DNS of a nonreacting mixing layer were used. ${ }^{22}$ The DNS was conducted with a high-order finite difference code that employs the low Mach number approximation. ${ }^{23}$ The flow was discretized by the use of a nonstaggered grid with dimensions of $481 \times 241 \times 39$ in the streamwise, transverse, and spanwise directions, respectively. The Reynolds number based on the inlet vorticity thickness and the freestream velocity difference was 200 .

The second set of data is from DNS for nonreacting channel and Couette flows (see Ref. 24). The grid size for both cases was $231 \times 200 \times 64$ points, and the Reynolds number based on the channel half-width was chosen to be 3000 for both flows. Temperature was taken as the passive scalar, and isothermal walls with a scaled temperature difference of one were used.

Note that, overall, the results obtained from the DNS data for Couette and channel flows were very similar to ones obtained from the mixing-layer DNS data; thus, only figures and statistics computed from the mixing-layer data are shown.

The shape of both base and test filters were defined to be triangular, ${ }^{18}$ and the models tested were taken from Eqs. (8) and (14) with $\theta_{\phi}$ calculated directly from the DNS results.

\section{A. SGS Scalar Flux}

Figures 1 and 2 show scatter plots of $\tau_{1, \phi}$ computed from the DNS data for the mixing layer vs the values of models computed from the filtered DNS field.

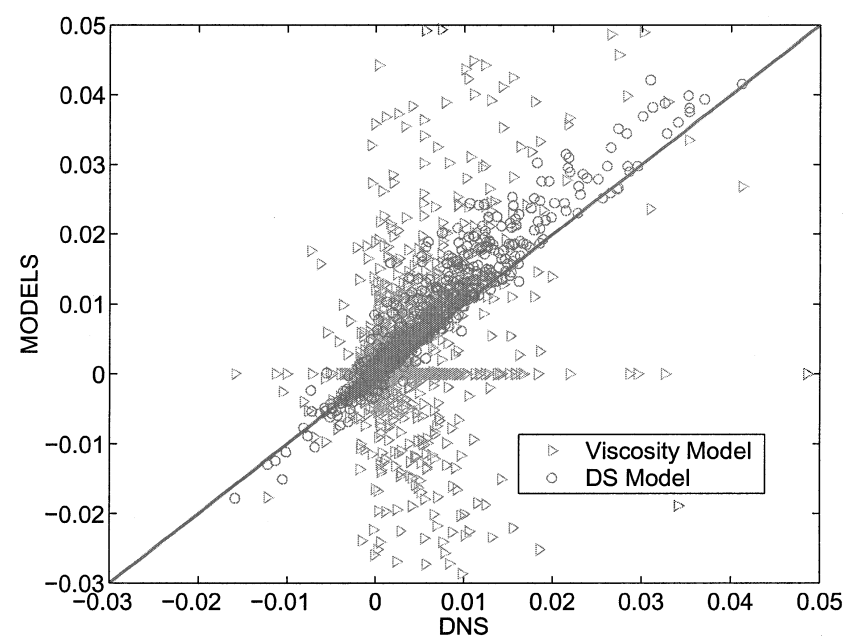

Fig. 1 Scatter plot of $\tau_{1, \phi}$ computed from DNS vs model for DS and viscosity models.

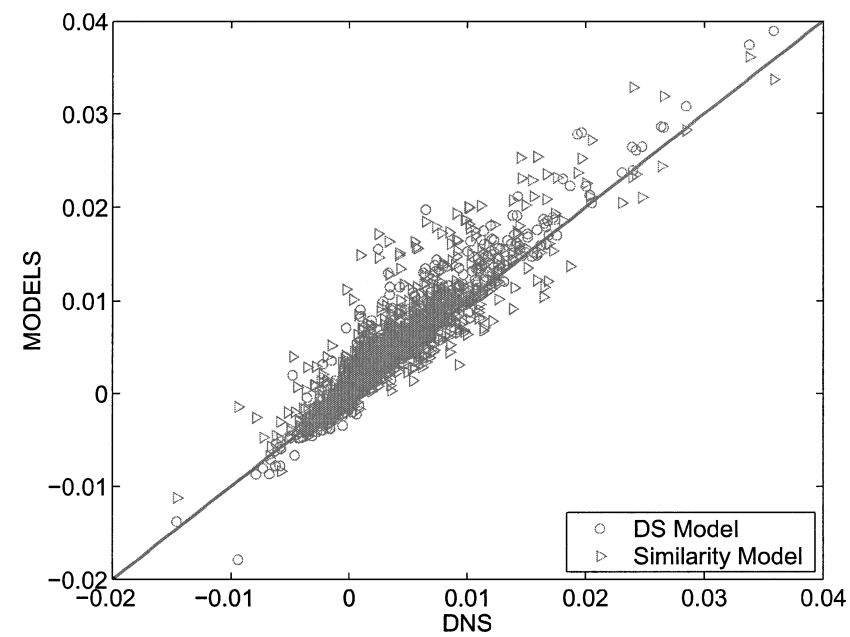

Fig. 2 Scatter plot of $\tau_{1, \phi}$ computed from DNS vs model for DS and similarity models. 
Figure 1 compares the DS model with the most popular viscositytype model $\tau_{i, \phi} \approx C_{s} \Delta^{2}\left|\bar{S}_{i j}\right| \partial \bar{\phi} / \partial x_{i}$, where $\bar{S}_{i j}=1 / 2\left(\partial \bar{u}_{i} / \partial x_{j}+\right.$ $\left.\partial \bar{u}_{j} / \partial x_{i}\right)$. Figure 2 compares the DS model with the similarity model $\tau_{i, \phi} \approx L_{1, \phi}$. The ratio of the base to test filter size for the viscosity and similarity models was chosen to obtain the peak performance of both models, whereas for the DS model, the test filter size was always equal to the base filter size. Namely, if we denote the DNS grid cell size by $\Delta_{g}$ in Fig. 1, for the viscosity model, $\Delta=8 \Delta_{g}$ and $\hat{\Delta}=14 \Delta_{g}$; for the DS model, $\hat{\Delta}=\Delta=8 \Delta_{g}$. In Fig. 2, for the similarity model, $\Delta=6 \Delta_{g}$ and $\hat{\Delta}=8 \Delta_{g}$; for the DS model, $\hat{\Delta}=\Delta=6 \Delta_{g}$.

From Fig. 1 it is clear that the assumption on which the viscosity model is built, that $\tau_{i \phi}$ scales well with $\Delta^{2}\left|S_{i j}\right| \partial \bar{\phi} / \partial x_{i}$, does not hold well. In the remainder of the paper, comparisons will be restricted to the DS and similarity models only.

Because no apparent difference between the two models could be seen on the scatter plot in Fig. 2, we employed an approach that seemed more informative, finding statistical characteristics of the relative error for a given model. The relative error is defined as

$$
E=\left[\left(\tau_{i, \phi}\right)_{\text {model }}-\tau_{i, \phi}\right] / \tau_{i, \phi}
$$

and is evaluated with the $\tau_{i, \phi}$ computed from the DNS field. Figure 3 shows the PDF for relative errors of the two models. From Fig. 3, the advantage of the DS model is apparent.

Figure 4 shows the results of evaluation of the DS and similarity models under more strenuous conditions, namely, a highly nonisotropic filter. The base filter dimensions were chosen to be $6 \Delta_{g} \times 14 \Delta_{g} \times 10 \Delta_{g}$. Again, different test filter sizes were employed for each model to capture the peak performance for the given base filter: $6 \Delta_{g} \times 14 \Delta_{g} \times 10 \Delta_{g}$ for the DS model and $10 \Delta_{g} \times 20 \Delta_{g} \times 16 \Delta_{g}$ for the similarity model. From Fig. 4, it is apparent that the DS model performs better than the similarity model.

In addition, we varied base and filter sizes to investigate the behavior of the DS and similarity models for different degrees of resolvness, filter skewness, and test to base filter size ratio. The PDF of relative error was found for every case, and statistical characteristics were computed. The results are listed in Tables 1 and 2 .

Both Tables 1 and 2 have 22 cases. The first 15 examine the behavior of the DS model with respect to the base filter size and the ratio of $\hat{\Delta}$ to $\Delta$. The last six rows deal with irregularly shaped filters

These results lead to a conclusion that the overall performance of the DS model seems very satisfactory compared to other known models. A traditional model (the similarity model) was evaluated as well, its performance being best for a ratio of $\hat{\Delta}$ to $\Delta$ of

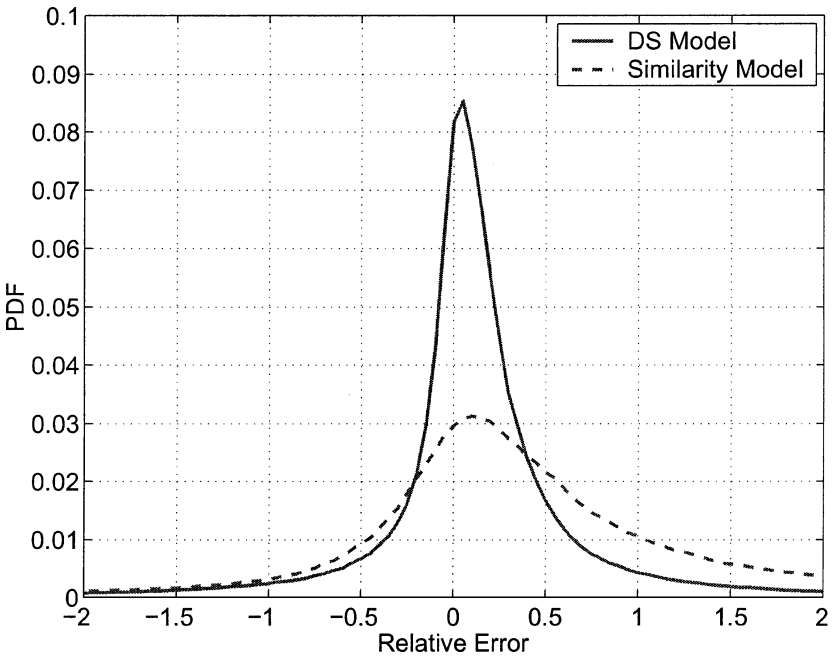

Fig. 3 PDFs of the relative errors for the DS and similarity models for the subgrid transport term $\tau_{1, \phi}$.

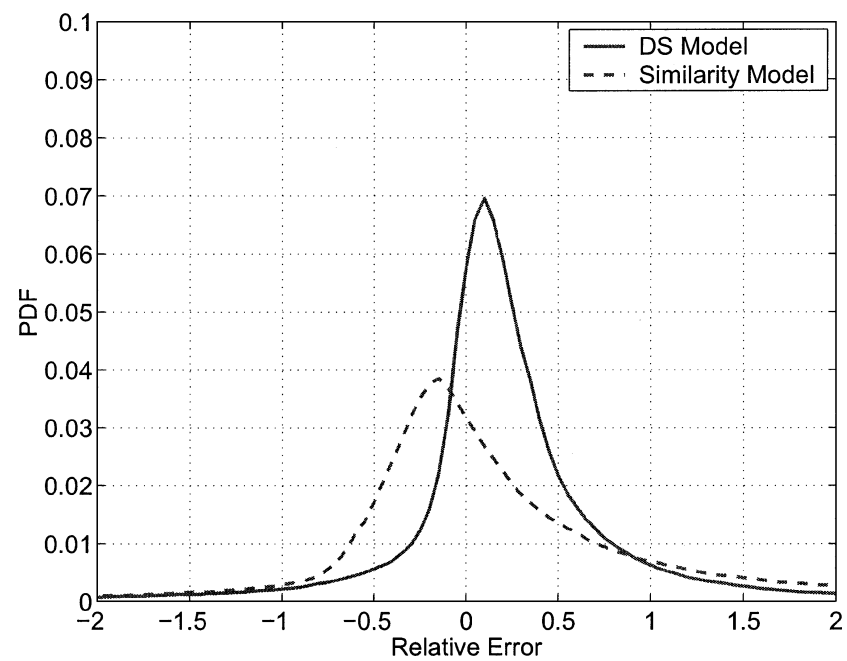

Fig. 4 PDFs of the relative errors for the DS and similarity models for the subgrid transport term $\tau_{1, \phi}$; filter shape corresponds to entry 20 in Table 1.

Table 1 Statistical characteristics of the PDF of relative error for the DS model (8) for $\tau_{1, \phi}$, mixing layer DNS data

\begin{tabular}{lccccr}
\hline \hline Run & Base & Test & Mean & Standard deviation & \multicolumn{1}{c}{ Median } \\
\hline 1 & $4 \times 4 \times 4$ & $4 \times 4 \times 4$ & $0.537 e-01$ & $0.992 e+00$ & $0.401 e-01$ \\
2 & $4 \times 4 \times 4$ & $6 \times 6 \times 6$ & $-0.199 e-01$ & $0.134 e+01$ & $0.746 e-02$ \\
3 & $4 \times 4 \times 4$ & $8 \times 8 \times 8$ & $-0.602 e-01$ & $0.179 e+01$ & $-0.278 e-01$ \\
4 & $4 \times 4 \times 4$ & $10 \times 10 \times 10$ & $-0.827 e-01$ & $0.223 e+01$ & $-0.615 e-01$ \\
5 & $4 \times 4 \times 4$ & $14 \times 14 \times 14$ & $-0.101 e+00$ & $0.292 e+01$ & $-0.116 e+00$ \\
6 & $6 \times 6 \times 6$ & $6 \times 6 \times 6$ & $0.124 e+00$ & $0.198 e+01$ & $0.883 e-01$ \\
7 & $6 \times 6 \times 6$ & $8 \times 8 \times 8$ & $0.797 e-01$ & $0.230 e+01$ & $0.756 e-01$ \\
8 & $6 \times 6 \times 6$ & $10 \times 10 \times 10$ & $0.543 e-01$ & $0.273 e+01$ & $0.584 e-01$ \\
9 & $6 \times 6 \times 6$ & $14 \times 14 \times 14$ & $0.281 e-01$ & $0.347 e+01$ & $0.198 e-01$ \\
10 & $8 \times 8 \times 8$ & $8 \times 8 \times 8$ & $0.178 e+00$ & $0.279 e+01$ & $0.123 e+00$ \\
11 & $8 \times 8 \times 8$ & $10 \times 10 \times 10$ & $0.147 e+00$ & $0.300 e+01$ & $0.119 e+00$ \\
12 & $8 \times 8 \times 8$ & $14 \times 14 \times 14$ & $0.109 e+00$ & $0.356 e+01$ & $0.982 e-01$ \\
13 & $10 \times 10 \times 10$ & $10 \times 10 \times 10$ & $0.203 e+00$ & $0.315 e+01$ & $0.141 e+00$ \\
14 & $10 \times 10 \times 10$ & $14 \times 14 \times 14$ & $0.156 e+00$ & $0.345 e+01$ & $0.134 e+00$ \\
15 & $14 \times 14 \times 14$ & $14 \times 14 \times 14$ & $0.174 e+00$ & $0.339 e+01$ & $0.146 e+00$ \\
16 & $6 \times 14 \times 6$ & $6 \times 14 \times 6$ & $0.196 e+00$ & $0.280 e+01$ & $0.151 e+00$ \\
17 & $6 \times 6 \times 14$ & $6 \times 6 \times 14$ & $0.150 e+00$ & $0.342 e+01$ & $0.635 e-01$ \\
18 & $14 \times 6 \times 6$ & $14 \times 6 \times 6$ & $0.184 e+00$ & $0.310 e+01$ & $0.130 e+00$ \\
19 & $6 \times 10 \times 14$ & $6 \times 10 \times 14$ & $0.171 e+00$ & $0.305 e+01$ & $0.105 e+00$ \\
20 & $6 \times 14 \times 10$ & $6 \times 14 \times 10$ & $0.222 e+00$ & $0.321 e+01$ & $0.162 e+00$ \\
21 & $14 \times 6 \times 10$ & $14 \times 6 \times 10$ & $0.183 e+00$ & $0.343 e+01$ & $0.131 e+00$ \\
22 & $6 \times 14 \times 10$ & $10 \times 20 \times 14$ & $0.145 e+00$ & $0.332 e+01$ & $0.127 e+00$ \\
\hline
\end{tabular}

${ }^{a}$ Base and test columns refer to the base and test filter sizes in terms of DNS grid cell size $\Delta_{g}$. 
Table 2 Statistical characteristics of the PDF of relative error for the similarity model for $\tau_{1, \phi}$, mixing layer DNS data ${ }^{a}$

\begin{tabular}{|c|c|c|c|c|c|}
\hline Run & Base & Test & Mean & Standard deviation & Median \\
\hline 1 & $4 \times 4 \times 4$ & $4 \times 4 \times 4$ & $-0.108 e+00$ & $0.747 e+00$ & $-0.120 e+00$ \\
\hline 2 & $4 \times 4 \times 4$ & $6 \times 6 \times 6$ & $0.131 e+01$ & $0.358 e+01$ & $0.106 e+01$ \\
\hline 3 & $4 \times 4 \times 4$ & $8 \times 8 \times 8$ & $0.307 e+01$ & $0.905 e+01$ & $0.225 e+01$ \\
\hline 4 & $4 \times 4 \times 4$ & $10 \times 10 \times 10$ & $0.497 e+01$ & $0.166 e+02$ & $0.332 e+01$ \\
\hline 5 & $4 \times 4 \times 4$ & $14 \times 14 \times 14$ & $0.862 e+01$ & $0.346 e+02$ & $0.505 e+01$ \\
\hline 6 & $6 \times 6 \times 6$ & $6 \times 6 \times 6$ & $-0.178 e+00$ & $0.132 e+01$ & $-0.228 e+00$ \\
\hline 7 & $6 \times 6 \times 6$ & $8 \times 8 \times 8$ & $0.530 e+00$ & $0.341 e+01$ & $0.293 e+00$ \\
\hline 8 & $6 \times 6 \times 6$ & $10 \times 10 \times 10$ & $0.138 e+01$ & $0.677 e+01$ & $0.803 e+00$ \\
\hline 9 & $6 \times 6 \times 6$ & $14 \times 14 \times 14$ & $0.326 e+01$ & $0.166 e+02$ & $0.167 e+01$ \\
\hline 10 & $8 \times 8 \times 8$ & $8 \times 8 \times 8$ & $-0.248 e+00$ & $0.144 e+01$ & $-0.318 e+00$ \\
\hline 11 & $8 \times 8 \times 8$ & $10 \times 10 \times 10$ & $0.201 e+00$ & $0.292 e+01$ & $-0.151 e-01$ \\
\hline 12 & $8 \times 8 \times 8$ & $14 \times 14 \times 14$ & $0.129 e+01$ & $0.802 e+01$ & $0.538 e+00$ \\
\hline 13 & $10 \times 10 \times 10$ & $10 \times 10 \times 10$ & $-0.321 e+00$ & $0.145 e+01$ & $-0.401 e+00$ \\
\hline 14 & $10 \times 10 \times 10$ & $14 \times 14 \times 14$ & $0.345 e+00$ & $0.401 e+01$ & $-0.266 e-01$ \\
\hline 15 & $14 \times 14 \times 14$ & $14 \times 14 \times 14$ & $-0.460 e+00$ & $0.122 e+01$ & $-0.562 e+00$ \\
\hline 16 & $6 \times 14 \times 6$ & $6 \times 14 \times 6$ & $-0.140 e+00$ & $0.172 e+01$ & $-0.262 e+00$ \\
\hline 17 & $6 \times 6 \times 14$ & $6 \times 6 \times 14$ & $-0.541 e+00$ & $0.101 e+01$ & $-0.582 e+00$ \\
\hline 18 & $14 \times 6 \times 6$ & $14 \times 6 \times 6$ & $-0.264 e+00$ & $0.146 e+01$ & $-0.319 e+00$ \\
\hline 19 & $6 \times 10 \times 14$ & $6 \times 10 \times 14$ & $-0.483 e+00$ & $0.108 e+01$ & $-0.558 e+00$ \\
\hline 20 & $6 \times 14 \times 10$ & $6 \times 14 \times 10$ & $-0.264 e+00$ & $0.157 e+01$ & $-0.379 e+00$ \\
\hline 21 & $14 \times 6 \times 10$ & $14 \times 6 \times 10$ & $-0.376 e+00$ & $0.143 e+01$ & $-0.432 e+00$ \\
\hline 22 & $6 \times 14 \times 10$ & $10 \times 20 \times 14$ & $0.613 e+00$ & $0.482 e+01$ & $0.535 e-01$ \\
\hline
\end{tabular}

${ }^{a}$ Base and test columns refer to the base and test filter sizes in terms of DNS grid cell size $\Delta_{g}$.

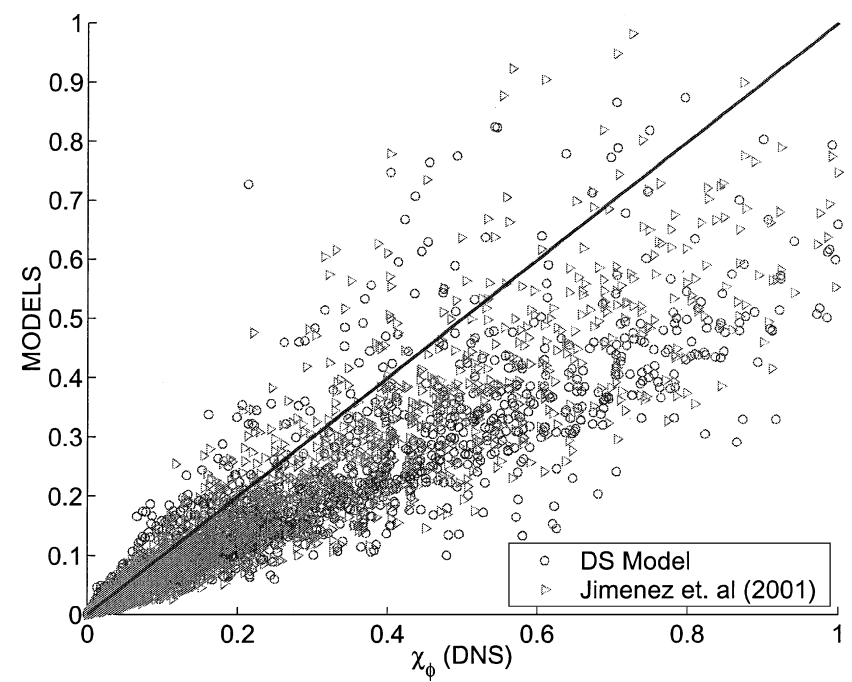

Fig. 5 Scatter plot of instantaneous values of $\chi_{\phi}$ and its models, DS model (14) and momentum-based model, ${ }^{12}$ for the case $\Delta=\hat{\Delta}=7 \Delta_{g}$, where $\Delta_{g}$ is the DNS grid cell size.

approximately 1.2. The peak performance of the DS model is observed for the cases of $\hat{\Delta}=\Delta$. Overall, for the DS model, the mean value of the relative error stays within $20 \%$, and the median stays within $15 \%$, even for the filters with large degree of skewness (the last six entries in Table 1). The similarity model does not exhibit such robust behavior, as can be seen from Table 2 .

\section{B. SGS Scalar Dissipation}

The DS model (14) for scalar dissipation (11) was compared to one of the recent LES models found in the literature ${ }^{12}$ :

$$
\bar{\chi} \equiv D \overline{\frac{\partial \phi}{\partial x_{i}} \frac{\partial \phi}{\partial x_{i}}} \approx C \cdot \frac{\epsilon}{k} \cdot \theta_{\phi}
$$

where $\epsilon=\overline{v\left(\partial u_{i} / \partial x_{j} \partial u_{i} / \partial x_{j}\right)}$ is the filtered kinetic energy dissipation rate. This model, although criticized in literature, was chosen for comparison due to its simplicity, relatively good performance, and nondependence on spectral formulation, which broadens the applicability range.

The results of the comparison are presented in Figs. 5 and 6, the scatter plot and the PDF of relative error, respectively. The

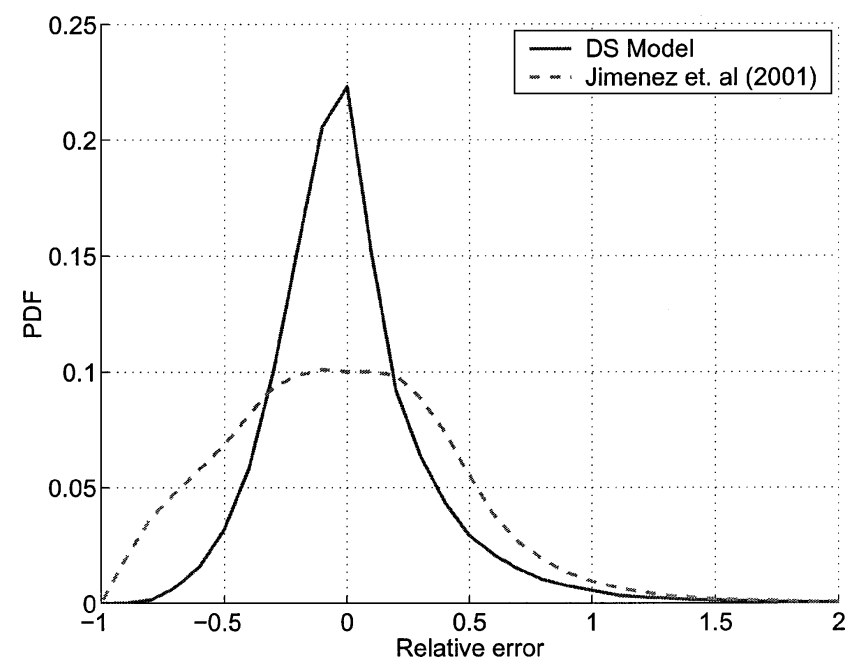

Fig. 6 PDF of relative error for the DS model for $\chi_{\phi}$ and the momentum-based model for $\chi_{\phi}{ }^{12}$; for the DS model, the base and test filter sizes are $\Delta=\hat{\Delta}=7 \Delta x$, and DNS data for nonreacting mixing layer was used.

quantity that was modeled by the use of both approaches was $\chi_{\phi}=\bar{\chi}+D\left(\partial \bar{\phi} / \partial x_{i} \partial \bar{\phi} / \partial x_{i}\right)$. Figure 5 does not seem to give any definite comparison, but from Fig. 6 , the advantage of DS model is clear.

To investigate the sensitivity of the DS model for scalar dissipation (14) to the ratio $\hat{\Delta} / \Delta$ and to the base filter size and shape, we computed the PDF of relative error for various cases. Similar to the DS model for the flux term, the peak performance of the model is observed for the ratio $\hat{\Delta} / \Delta$ close to 1 , and the mean relative error stayed within $20 \%$.

\section{A Posteriori Evaluation}

Although the a priori test is significant, a more complete a posteriori test should be administered to validate the model's capability of predicting a time-evolving flow. Thus, a comparison of the DNS and LES is made for the case of a nonreacting incompressible mixing layer.

The DNS validation case was simulated with a finite difference nondissipative code that is 11th-order accurate in space and fifth-order accurate in time. A thorough accuracy study was 
Table $3 L^{2}$ norm of error in the velocity field for three different orders of accuracy ${ }^{22}$

\begin{tabular}{lccc}
\hline \hline$N^{\mathrm{a}}$ & 3rd-order & 5th-order & 7th-order \\
\hline 30 & $1.0 e-2$ & $1.0 e-3$ & $2.0 e-4$ \\
60 & $1.5 e-3$ & $2.0 e-5$ & $1.5 e-6$ \\
90 & $1.2 e-4$ & $1.5 e-6$ & $3.0 e-7$ \\
\hline
\end{tabular}

${ }^{\mathrm{a}} N$ is number of grid points in each direction.

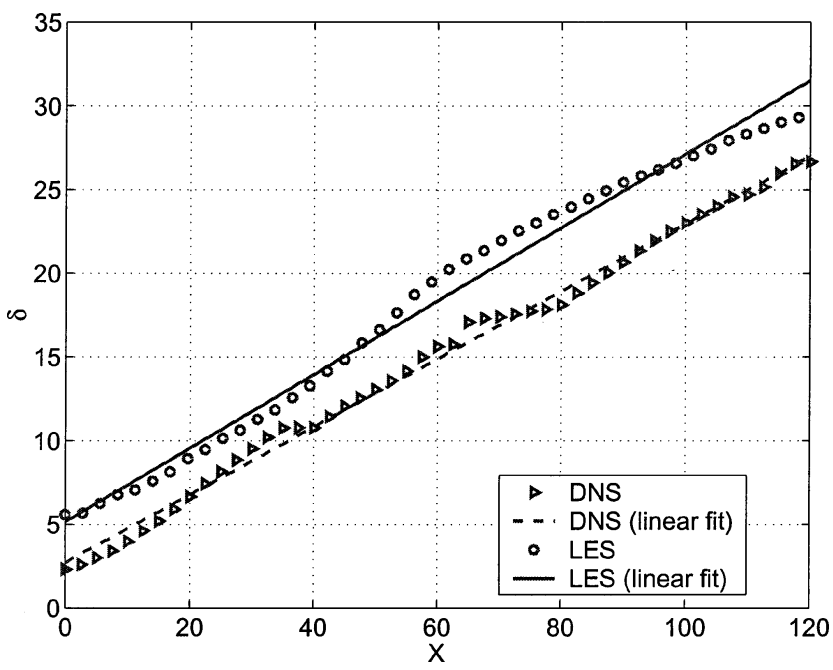

Fig. 7 Comparison of the $1 \%$ scalar difference mixing-layer width obtained from DNS ${ }^{22}$ and LES runs.

performed by comparison of the velocity field to an analytical solution for evolution of Taylor vortex in uniform flow with $R e=10^{6}$ (Ref. 22). A brief summary of validation results is given in the Table 3. Overall, the desired accuracy is maintained for each grid density.

An LES of a nonreacting incompressible mixing layer was performed with the same setup as the DNS, and the same DNS code adapted for LES calculations. The grid spacing was chosen to be roughly four times larger than the DNS in each direction. The base filter size, however, did not coincide with the grid cell size, but the characteristic length of the base filter was chosen to be $4 \Delta_{g}$, where $\Delta_{g}$ is the LES grid cell size. That was done to minimize the finite differencing LES error while keeping the computational cost bearable. ${ }^{25}$ The test filter size was equal to the base filter size, that is, $\hat{\Delta}=\Delta$.

Figure 7 presents the rate of growth of the mixing layer found with the $1 \%$ scalar difference found from DNS and LES. The growth rate in LES was found to be close to linear, as predicted by DNS and generally found in the literature..$^{22,26,27}$ LES and DNS mixing layer widths demonstrated by Fig. 7 have the same linear growth rate and differ only by the placement of virtual origin.

The following expression can be taken as an approximation for the growth rate ${ }^{26}$ :

$$
\delta / x=C_{\delta}[(1-r) /(1+r)]
$$

where $\delta$ is the $1 \%$ passive scalar thickness, $x$ is the distance downstream, $r$ is the velocity ratio, and $C_{\delta}$ is the growth rate constant. The value of $C_{\delta}$ has been found to be in the range of $0.25, \ldots, 0.45$ by a collection of experimental studies. ${ }^{22}$ Given the current velocity ratio $r=1 / 3$, the approximate value of $C_{\delta}$ computed from the LES is 0.4554 , which belongs to the admissible range. The DNS prediction of the value of $C_{\delta}$ was 0.422 .

The examination of the self-similarity of the scalar field in the mixing layer is presented in Figs. 8 and 9. The profiles of the scalar mean and rms values were computed at various distances downstream and then plotted vs the self-similarity variable $\eta=(y-L / 2) / \delta$, where $y$ is the transverse coordinate, $L$ is the domain width in the transverse direction, and $\delta$ is the $1 \%$ pas-

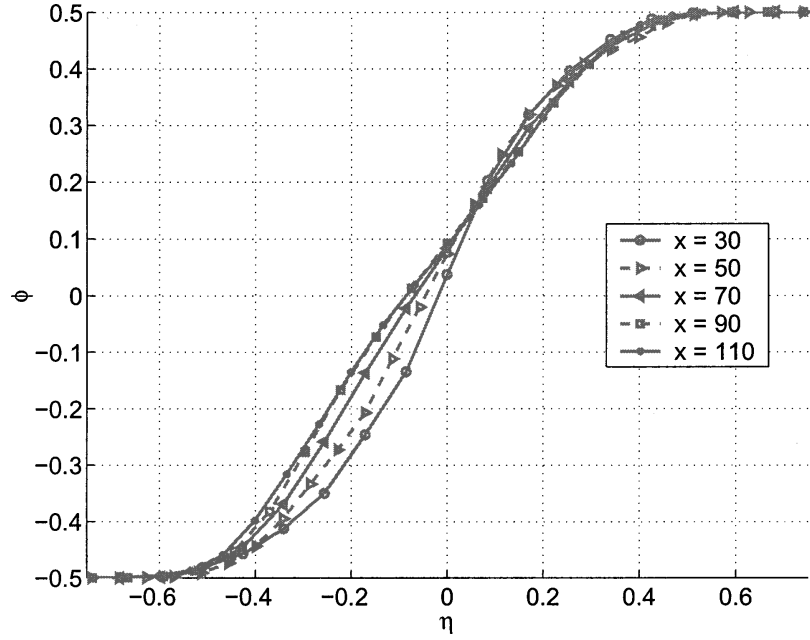

Fig. 8 Self-similarity of the mean scalar field in the LES mixing layer.

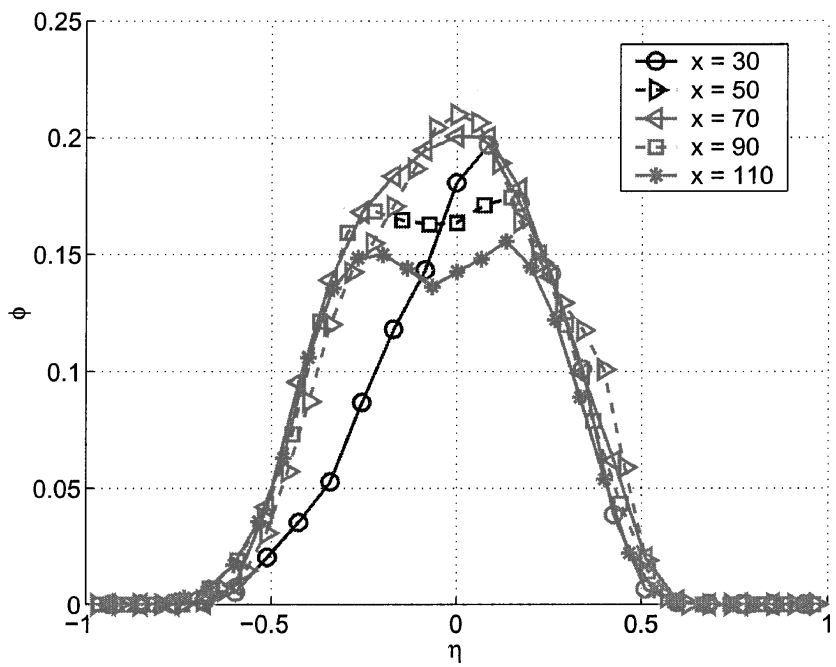

Fig. 9 Self-similarity of the RMS value of the scalar field in the LES mixing layer

sive scalar thickness defined earlier. As can be seen from Figs. 8 and 9, neither mean nor rms values exhibit the self-similar behavior that indicates that the mixing layer is not yet fully developed and is still in transition. This agrees well with the DNS results. ${ }^{22}$

We compared the mean temperature profiles near the end of the computational domain (Fig. 10). Note that both profiles have a small but distinguishable bump slightly below the center of the computational domain. This distortion in the mean temperature profile indicates that the center of the layer is better mixed by the spanwise vortical structures. This gives a more homogeneous temperature in the center of the layer. Similar phenomena were observed in experimental work ${ }^{27}$ where a scalar profile like this was seen as an indication of a nonmarching PDF.

Finally, we compared the variance of the scalar as a function of $y$, the transverse coordinate. This is done by fixing the $x$ (streamwise) coordinate, finding the variance $\operatorname{var}(\phi) \equiv\left\langle\phi^{2}\right\rangle-\langle\phi\rangle^{2}$, and averaging it in the $z$ (spanwise) direction. The angle brackets indicate the ensemble averaging.

The quantities compared in the Fig. 11 are $\overline{\operatorname{var}(\phi)}=\langle\overline{\phi \phi}\rangle-$ $\overline{\langle\phi\rangle\langle\phi\rangle}$ obtained from the DNS data for the mixing layer, $\operatorname{var}(\bar{\phi})$ obtained from the LES data, and $\operatorname{var}(\bar{\phi})+2\left\langle\theta_{\phi}\right\rangle=\langle\overline{\phi \phi}\rangle-\langle\bar{\phi}\rangle\langle\bar{\phi}\rangle$ obtained from LES data. The last quantity is assumed to be close to $\overline{\operatorname{var}(\phi)}$, that is, the difference between $\overline{\langle\phi\rangle\langle\phi\rangle}$ and $\langle\bar{\phi}\rangle\langle\bar{\phi}\rangle$ is assumed to be small.

The $x$ (streamwise) coordinate was different for DNS and LES statistics: $x=110$ for DNS and $x=100$ for LES. This was motivated 


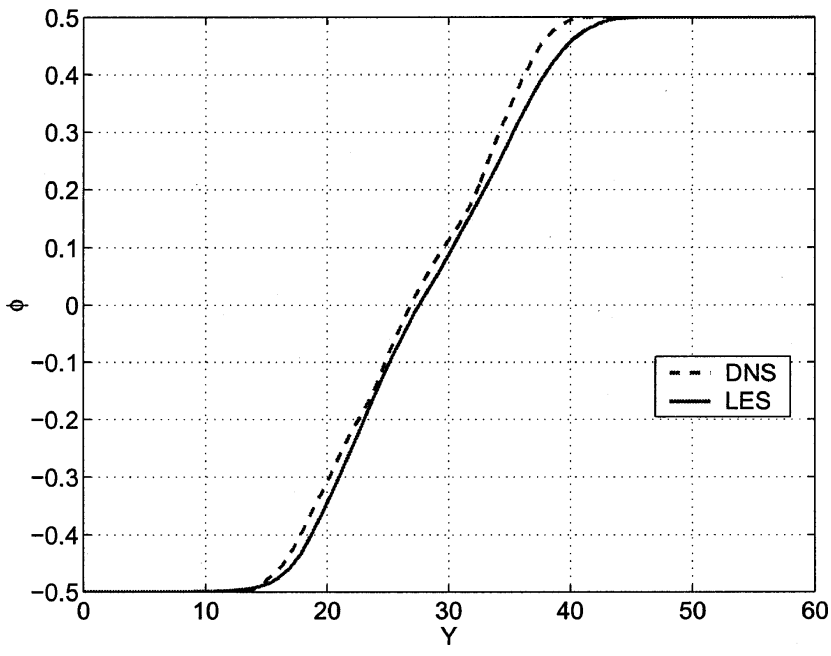

Fig. 10 Comparison of the mean scalar profile at $x=110$ between DNS and LES results.

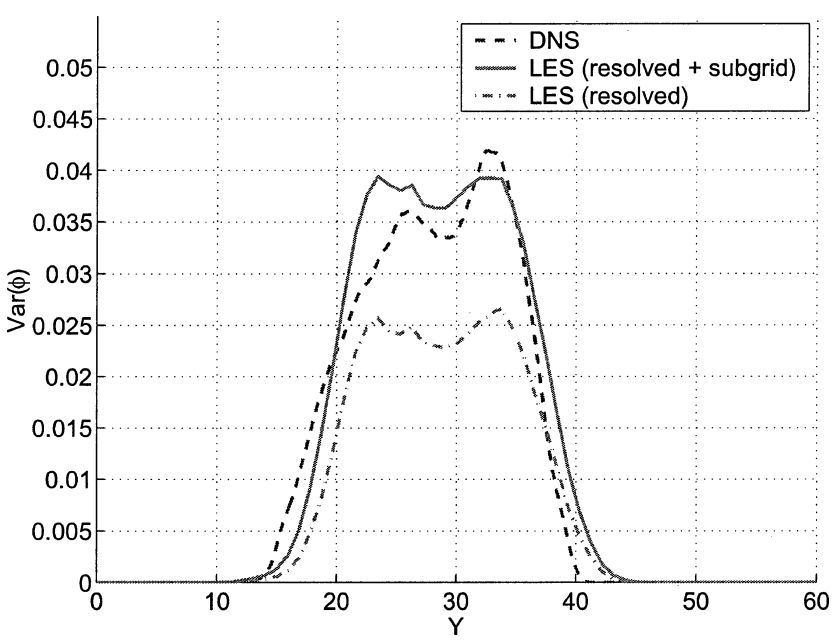

Fig. 11 Variance of the scalar as a function of transverse coordinate; comparison between LES and DNS results.

by the fact that the distance between the virtual origins of DNS and LES shear layers was found to be approximately 10 units. Virtual origins were found with the linear fits for growth rates displayed in Fig. 7.

From Fig. 11, it is apparent that the comparison between DNS and LES results is good. Moreover, Fig. 11 demonstrates the importance of the subgrid scalar variance. Indeed, it contributed as much as 40 $45 \%$ to the total scalar variance.

Overall, the agreement of the LES and DNS data was found to be good. The LES predicted the scalar field characteristics well.

\section{Conclusions}

Two new models for the LES subgrid scalar flux term and the subgrid scalar dissipation were proposed. The models are considered to be of a new class of LES models, namely, dynamic structure (DS) models. The models were evaluated with DNS data and found to represent the effects of the subgrid scalar field on the resolved scalar field adequately. Also, models found in the literature were evaluated by the use of the same set of data for the purpose of comparison to the DS models. The DS models were found to perform better.

To evaluate the DS models a posteriori, an LES of a nonreacting mixing layer was performed with both models, and the results showed a good agreement with the DNS run performed for the same setup. The subgrid scalar variance was found to contribute a significant part to the total scalar variance found from DNS data.
The DS models seem to perform well under various conditions, including different base filter sizes, various degrees of skewness, and ratios of the base to the test filter sizes. The peak performance of the DS models was observed for the case when base and test filter sizes were equal.

\section{Acknowledgments}

This work was sponsored in part by the U.S. Army Research Office under Contract/Grant DAAD19-00-1-0487 and the U.S. Air Force Office of Scientific Research under Grant F49620-02-1-0348.

\section{References}

${ }^{1}$ Meneveau, C., and Katz, J., "Scale-Invariance and Turbulent Models for Large-Eddy Simulation," Annual Review in Fluid Mechanics, Vol. 32, 2000, pp. 1-32.

${ }^{2}$ Cook, A. W., and Riley, J. J., "A Subgrid Model for Equilibrium Chemistry in Turbulent Flows," Physics of Fluids, Vol. 6, 1994, p. 2868.

${ }^{3}$ Cook, A. W., and Riley, J. J., "Subgrid-Scale Modeling for Turbulent Reacting Flows," Combustion and Flame, Vol. 112, 1998, pp. 593-606.

${ }^{4}$ Smagorinsky, J., "General Circulation Experiments with the Primitive Equations I. The Basic Experiment," Monthly Weather Review, Vol.91, 1963, pp. $99-165$.

${ }^{5}$ Cabot, W., and Moin, P., "Large Eddy Simulation of Scalar Transport with the Dynamic Subgrid-Scale Model," Center for Turbulence Research, Manuscript 128, NASA Ames Research Center, 1991.

${ }^{6}$ Moin, P., Squires, K., Cabot, W., and Lee, S., "A Dynamic Subgrid-Scale Model for Compressible Turbulence and Scalar Transport," Physics of Fluids A, Vol. 3, No. 11, 1991, pp. 2746-2757.

${ }^{7}$ Germano, M., Piomelli, U., Moin P., and Cabot, W. H., "A Dynamic Subgrid-Scale Eddy Viscosity Model," Physics of Fluids A, Vol. 3, 1991, p. 1760 .

${ }^{8}$ Bardina, J., Ferziger, J. H., and Reynolds, W. C., "Improved Subgrid Scale Models for Large-Eddy Simulation," AIAA Paper 80-1357, 1980.

${ }^{9}$ Speziale, C. G., Eriebacher, G., Zang, T. A., and Hussaini, M. Y., "The Subgrid-Scale Modeling of Compressible Turbulence," Physics of Fluids, Vol. 31, No. 4, 1998, p. 940.

${ }^{10}$ Bardina, J., "Improved Turbulence Models Based on Large Eddy Simulation of Homogeneous, Incompressible, Turbulent Flows," Ph.D. Dissertation, Dept. of Mechanical Engineering, Stanford Univ., Stanford, CA, Nov. 1983.

${ }^{11}$ Adumitroaie, V., Frankel, S. H., Madina, C. K., and Givi, P., "LargeEddy Simulation and Direct Numerical Simulation of High Speed Turbulent Reacting Flows," Turbulence Research Lab. Rept., State Univ. of New York, Buffalo, NY, 1993.

${ }^{12}$ Jimenez, C., Ducros, F., Cuenot, B., and Bedat, B., "Subgrid Scale Variance and Dissipation of a Scalar Field in Large Eddy Simulation," Physics of Fluids, Vol. 13, No. 6, 2002, pp. 1748-1754.

${ }^{13}$ Girimaji, S., and Zhou, Y., "Analysis and Modeling of Subgrid Scalar Mixing Using Numerical Data," Physics of Fluids, Vol. 8, No. 5, 1996, p. 1224.

${ }^{14}$ de Bryun Kops, S. M., Riley, J. J., Kosaly, G., and Cook, A. W., "Investigation of Modeling for Non-Premixed Turbulent Combustion," Flow, Turbulence and Combustion, Vol. 60, 1998, p. 105.

${ }^{15}$ Cook, A. W., and Bushe, W. K., "A Subgrid-Scale Model for the Scalar Dissipation Rate in Non-Premixed Combustion," Physics of Fluids, Vol. 11, No. 3, 1999, p. 746.

${ }^{16}$ Liu, S., Meneveau, C., and Katz, J., "On the Properties of Similarity Subgrid-Scale Models as Deduced from Measurements in a Turbulent Jet," Journal of Fluid Mechanics, Vol. 275, 1994, p. 83.

${ }^{17} \mathrm{Gao}, \mathrm{F}$., and O'Brien, E. E., "A Large Eddy Simulation Scheme for Turbulent Reacting Flows," Physics of Fluids A, Vol. 5, 1993, p. 1282 .

${ }^{18}$ Pomraning, E., and Rutland, C. J., "Dynamic One-Equation Nonviscosity Large-Eddy Simulation Model," AIAA Journal, Vol. 40, No. 4, 2002 , pp. 689-701.

${ }^{19}$ Cook, A. W., "Determination of the Constant Coefficient in Scale Similarity Models of Turbulence," Physics of Fluids, Vol. 9, No. 5, 1997, pp. 1485-1487.

${ }^{20}$ Pierce, C., and Moin, P., “A Dynamic Model for Subgrid-Scale Variance and Dissipation Rate of a Conserved Scalar," Physics of Fluids, Vol. 10, No. 12, 1998, pp. 3041-3044.

${ }^{21}$ Yeo, W. K., "A Generalized High Pass/Low Pass Averaging Procedure for Deriving and Solving Turbulent Flow Equations," Ph.D. Dissertation, Dept. of Civil Engineering, The Ohio State Univ., Columbus, OH, 1987.

${ }^{22}$ Mason, S., "Turbulence Transport in Spatially Developing Reacting Shear Layers," Ph.D. Dissertation, Dept. of Mechanical Engineering, Univ. of Wisconsin, Madison, WI, 2000. 
${ }^{23}$ Rutland, C. J., Ferziger, J. H., and Cantwell, B. J., "Effects of Strain, Vorticity, and Turbulence on Premixed Flames," Thermosciences Div., Rept. TF-44, Dept. of Mechanical Engineering, Stanford Univ., Stanford, CA, 1989.

${ }^{24}$ Debusschere, B., "Turbulent Scalar Transport in Non-Reacting and Reacting Flows," Ph.D. Dissertation, Dept. of Mechanical Engineering, Univ. of Wisconsin, Madison, WI, 2001.

${ }^{25}$ Ghosal, S., "An Analysis of Numerical Errors in Large-Eddy Simulations of Turbulence," Journal of Computational Physics, Vol. 125, 1996, pp. 187-206.
${ }^{26}$ Dimotakis, P. E., "Turbulent Free Shear Layer Mixing and Combustion," High Speed Flight Propulsion Systems, edited by S. N. Murphy and E. T. Curran, Vol. 137, Progress in Astronautics and Aeronautics, AIAA, Washington, DC, 1991, pp. 265-340.

${ }^{27}$ Pickett, L. M., "Structure of a Planar Reacting Shear Layer Using Hydrocarbon Fuels," Ph.D. Dissertation, Dept. of Mechanical Engineering, Univ. of Wisconsin, Madison, WI, 2000.

P. Givi Associate Editor

\section{Orbital Mechanics, Third Edition}

\section{Vladimir A. Chobotov • The Aerospace Corporation}

Designed to be used as a graduate student textbook and a ready reference for the busy professional, this third edition of Orbital Mechanics is structured to allow you to look up the things you need to know. This edition includes more recent developments in space exploration (e.g. Galileo,

Cassini, Mars Odyssey missions). Also, the chapter on space debris was rewritten to reflect new developments in that area.

The well-organized chapters cover every basic aspect of orbital mechanics, from celestial relationships to the problems of space debris. The book is clearly written in language familiar to aerospace professionals and graduate students, with all of the equations, diagrams, and graphs you would like to have close at hand.

An updated software package on CD-ROM includes: HW Solutions, which presents a range of viewpoints and guidelines for solving selected problems in the text; Orbital Calculator, which provides an interactive environment for the generation of Keplerian orbits, orbital transfer maneuvers, and animation of ellipses, hyperbolas, and interplanetary orbits; and Orbital Mechanies Solutions.

- Basic Concepts

- Celestial Relationships

O

- Keplerian Orbits

- Position and Velocity as a Function of Time

- Orbital Maneuvers

- Complications to Impulsive Maneuvers

- Relative Motion in Orbit

- Introduction to Orbit Perturbations
- Orbit Perturbations: Mathematical Foundations

- Applications of Orbit Perturbations

- Orbital Systems

- Lunar and Interplanetary Trajectories

- Space Debris

- Optimal Low-Thrust Orbit Transfers

- Orbital Coverage

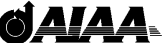

American Institute of Aeronautics and Astronautics Publications Customer Service, P.0. Box 960, Herndon, VA 20172-0960 Fax: 703/661-1501•Phone: 800/682-2422•E-Mail: warehouse@aiaa.org Order 24 hours a day at www.aiaa.org
2002, 460 pages, Hardback, with Software

ISBN: 1-56347-537-5

List Price: $\$ 100.95$ - AIAA Member Price: $\$ 69.95$ 